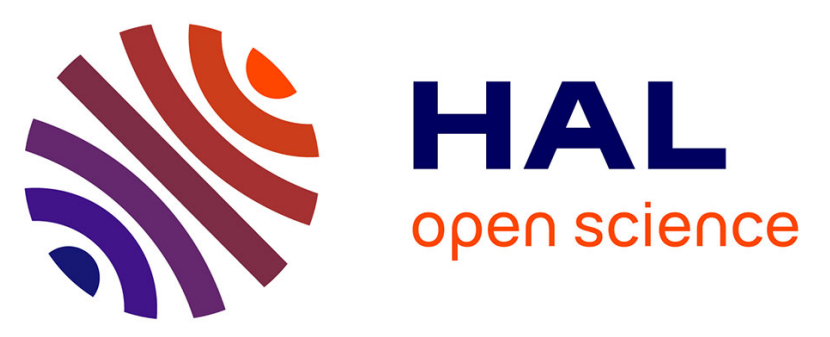

\title{
Modification des processus hors équilibre au cours de l'interaction entre un plasma basse pression et une surface. I. Cas d'un plasma de NH3. Application à la catalyse chimique
}

\author{
M. P. Bergougnan, A. Gicquel, J. Amouroux
}

\section{To cite this version:}

M. P. Bergougnan, A. Gicquel, J. Amouroux. Modification des processus hors équilibre au cours de l'interaction entre un plasma basse pression et une surface. I. Cas d'un plasma de NH3. Application à la catalyse chimique. Revue de Physique Appliquée, 1983, 18 (6), pp.335-346. 10.1051/rphysap:01983001806033500 . jpa-00245100

\author{
HAL Id: jpa-00245100 \\ https://hal.science/jpa-00245100
}

Submitted on 1 Jan 1983

HAL is a multi-disciplinary open access archive for the deposit and dissemination of scientific research documents, whether they are published or not. The documents may come from teaching and research institutions in France or abroad, or from public or private research centers.
L'archive ouverte pluridisciplinaire HAL, est destinée au dépôt et à la diffusion de documents scientifiques de niveau recherche, publiés ou non, émanant des établissements d'enseignement et de recherche français ou étrangers, des laboratoires publics ou privés. 


\title{
Modification des processus hors équilibre au cours de l'interaction entre un plasma basse pression et une surface. I. Cas d'un plasma de $\mathbf{N H}_{3}$. Application à la catalyse chimique
}

\author{
M. P. Bergougnan, A. Gicquel et J. Amouroux \\ Ecole Nationale Supérieure de Chimie de Paris, Equipe Réacteurs Chimiques en Phase Plasma, \\ 11, rue Pierre et Marie Curie, 75231 Paris Cedex 05, France
}

(Reçu le 19 janvier 1983, révisé le 24 février, accepté le 3 mars 1983)

\begin{abstract}
Résumé. - Dans cette étude nous avons mis en évidence les relations existant entre les processus de catalyse ou d'inhibition, le caractère hors équilibre du plasma et les transferts d'énergie et de matière, au cours de l'interaction entre un plasma $\mathrm{NH}_{3}$ basse pression et une surface.

Nous avons établi que l'activité catalytique ou inhibitrice d'un matériau est liée à son aptitude à modifier la répartition de l'énergie vibrationnelle du plasma dans la couche limite.

L'ensemble de nos résultats expérimentaux, les analyses spectroscopiques et de surface, ont mis en évidence une réaction de nitruration des surfaces reliée à la chimisorption dissociative des molécules.

Tout matériau qui augmente le caractère hors équilibre du plasma, favorise un processus catalytique et facilite l'étape de nitruration.

Tandis que tout matériau qui thermalise le plasma, inhibe la réaction de décomposition et défavorise l'étape de nitruration.
\end{abstract}

\begin{abstract}
The experimental study of the interaction between a low pressure $\mathrm{NH}_{3}$ plasma and a surface has pointed out the modification of the non equilibrium processes.

In this way, we have established the correlation between the heat and mass transfers in the boundary layer, the non equilibrium character of the plasma and the catalysis or inhibition process.

The main results have been obtained by using a spectroscopic analysis of the boundary layer, in order to measure the vibrational and rotational levels in function of the nature of the material. These results point out that the nitriding reaction of the solid surface (ESCA measurements) is correlated with the vibrational level of the $\mathrm{N}_{2}$ molecule and the temperature of the solid. This conclusion is in agreement with the following result : the $\mathrm{NH}_{3}$ decomposition rate increases with the vibrational $\mathrm{N}_{2}$ temperature and the nitriding of the surface.

So we can conclude that the catalytic or inhibitor effect during the interaction plasma-surface is controlled at first, by the energy balance between the boundary layer and the surface of the material, and in second by the dissociative chemisorption of the reactive species during the mass transfer.

Any material which increases the non equilibrium character of the plasma favours a catalytic process and makes easy the nitriding step.
\end{abstract}

\section{Introduction.}

Le milieu plasma basse pression est un milieu hors d'équilibre au sein duquel l'énergie n'est pas répartie de façon homogène entre les différents degrés de liberté de la molécule (vibration, rotation, translation).

Les cinétiques chimiques des milieux hors d'équilibre ont été étudiées, d'une part en phase gaz sur des faisceaux moléculaires par Polanyi [1-3], d'autre part en phase hétérogène à partir de modèles théoriques mathématiques par Mac Creery et Wolken $[4,5]$.

Un premier travail nous a permis d'établir des relations qualitatives entre le caractère hors d'équilibre du plasma basse pression et sa réactivité chi- mique en phase gaz et hétérogène $[6,7]$ pour des réactions de synthèse (HCN, NO) ou de décomposition $\left(\mathrm{NH}_{3}\right)$.

Nous avons développé l'application de ces théories à une réaction endothermique : la réaction de décomposition de $\mathrm{NH}_{3}$ en $\mathrm{N}_{2}+\mathrm{H}_{2}$. L'étude de ce processus en décharge H.F. basse pression présente plusieurs avantages : d'une part il est possible de suivre la plupart des espèces gazeuses par les processus d'émission qui caractérisent ses différents états électroniques, d'autre part toute modification énergétique due aux interactions plasma-surface peut être à nouveau caractérisée par l'émission de raies. L'étude en phase 
gaz de la réaction de décomposition de $\mathrm{NH}_{3}$ [8] a permis de mettre en évidence que l'étape clé de la réaction $\mathrm{NH}_{3} \rightarrow \mathrm{NH}+\mathrm{H}_{2}$, étape endothermique, est favorisée par une forte excitation vibrationnelle des molécules $\mathrm{NH}_{3}$. Ce résultat est en accord avec les travaux de Polanyi qui ont démontré que d'une façon générale les réactions endothermiques sont favorisées par une forte énergie vibrationnelle des réactifs, tandis que les réactions exothermiques le sont par une forte énergie translationnelle des réactifs [1-3].

Nous avons poursuivi notre travail par l'étude en phase hétérogène de la réaction de décomposition de $\mathrm{NH}_{3}$ afin d'établir les mécanismes réactionnels des interactions plasma-surface. A partir de l'étude parallèle de l'analyse spectroscopique du plasma, de la réactivité chimique, des mesures de température des solides introduits dans le réacteur, des mesures de surface (ESCA et micro-dureté) et en nous reposant sur les calculs théoriques de Mac Creery et Wolken $[4,5]$ et les travaux de Halpern et Rosner [9] dont nous avons vérifié les conclusions, nous avons pu correler l'évolution du taux de conversion de la réaction, les transferts énergétiques et de matière, avec le caractère hors d'équilibre du milieu. En effet, un matériau introduit dans un réacteur à plasma $\mathrm{NH}_{3}$ basse pression se comporte soit comme un catalyseur soit comme un inhibiteur de la réaction de décomposition de $\mathrm{NH}_{3}$.

L'activité catalytique ou inhibitrice de ce matériau est directement liée à l'aptitude de son nitrure (dont la formation sur quelques couches est instantanée) à modifier près de sa paroi (couche limite) la répartition énergétique du plasma, c'est-à-dire plus exactement à modifier l'énergie vibrationnelle qui est la forme d'énergie qui contrôle la réaction.

\section{Dispositif expérimental.}

Afin d'étudier la réaction de décomposition de $\mathrm{NH}_{3}$, nous avons utilisé un réacteur $(\varnothing=0,1 \mathrm{~m}, L=0,4 \mathrm{~m})$ chimique à plasma (Fig. 1). Un plasma basse pression $(P=1$ à 25 mbars) est généré par une décharge inductive H.F. (40 MHz). A une extrémité du réacteur,

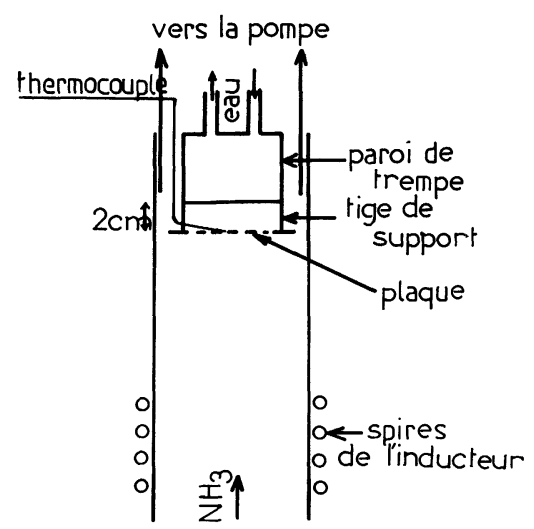

Fig. 1. - Schéma du réacteur à plasma basse pression.

[Scheme of the low pressure plasma reactor.] le plasma est trempé par une surface de cuivre refroidie à l'eau.

Le débit de gaz $\left(\mathrm{NH}_{3}\right)$ est $D^{0}=0,6 \mathrm{l} / \mathrm{min}$. et la puissance induite aux bornes de l'inducteur est $P_{\mathrm{I}}=1 \mathrm{~kW}$.

Une surface solide $\left(70 \times 50 \times 0,25 \mathrm{~mm}^{3}\right)$ de haute pureté $(99,9 \%)$ est introduite dans la torche à $2 \mathrm{~cm}$ de la trempe. Les matériaux utilisés sont soit des métaux de transition (W, Mo, Co) soit un semiconducteur (Si mono $\langle 100\rangle)$.

\section{Méthodes d'analyse (Fig. 2).}

La mesure et la quantification des processus rapides emploient la spectroscopie d'émission UV-visible. Cette méthode d'analyse permet de suivre in situ la réactivité et la trajectoire des espèces présentes dans le plasma et permet d'établir le bilan énergétique spécifique des molécules. Les transitions observées dans un plasma $\mathrm{NH}_{3}$ ont été longuement détaillées dans deux articles récents $[8,10]$.

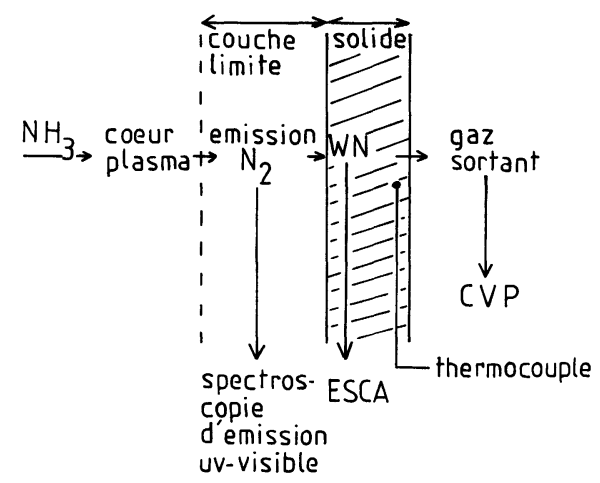

Fig. 2. - Méthodes d'analyse.

[Analysis methods.]

Nous rappellerons que de nombreuses espèces gazeuses sont détectables par spectroscopie d'émission UV-visible. Toutefois, nous avons choisi de suivre les caractéristiques de la molécule $\mathrm{N}_{2}$ pour étudier l'évolution de la transformation chimique. En effet, l'intensité lumineuse des transitions des différents états de cette molécule est très forte, et de plus les différents états électroniques de la molécule $\mathrm{N}_{2}$ sont très bien connus. La molécule $\mathrm{N}_{2}$ est donc un véritable traceur de la réaction. Par exemple, la température de vibration a été mesurée sur les bandes de transition $\Delta v=2$ du $2^{e}$ système positif de $\mathrm{N}_{2}$. (Transition $\mathrm{C}^{3} \Pi_{\mathrm{u}} \rightarrow \mathrm{B}^{3} \Pi_{\mathrm{g}}$ )

Le dispositif expérimental d'analyse spectroscopique est un monochromateur Jobin Yvon JY 38 de distance focale $1 \mathrm{~m}$, muni d'un réseau de 3600 traits/ $\mathrm{mn}$, thermorégulé à $35^{\circ} \mathrm{C}$ et comprenant un photomultiplicateur Hamamatsu R 446 pour analyser le rayonnement ultra-violet compris entre 3000 et $4500 \AA$

Les méthodes de mesure des processus lents sont les suivants : 
- La chromatographie en phase vapeur (CVP) permet d'établir le bilan matière selon la relation

$$
X=\frac{\left(\mathrm{NH}_{3}\right)^{0}-\left(\mathrm{NH}_{3}\right)}{\left(\mathrm{NH}_{3}\right)^{0}},
$$

où $X$ représente le taux de décomposition de $\mathrm{NH}_{3}$.

- Des thermocouples chromel-alumel blindés inox mesurent la température des surfaces solides et permettent d'établir le bilan énergétique.

- L'analyse ESCA des solides rend compte des modifications chimiques de la surface. Ces analyses ont été effectuées sur un appareil AEI type ES.200 B en mode FAT 65.

- Les mesures de microdureté, réalisées à l'aide d'une pointe diamant Vickers sous des charges de $50 \mathrm{~g}$ à $500 \mathrm{~g}$ indiquent la modification du réseau cristallin et permettent de déterminer l'épaisseur de la couche formée (variation des valeurs de microdureté en fonction de la profondeur de pénétration).

\section{Résultats expérimentaux.}

Notre démarche a consisté à analyser les phénomènes macroscopiques et microscopiques selon la procédure suivante.

En premier, nous avons déterminé le taux de décomposition de $\mathrm{NH}_{3}$ par analyse CVP des gaz sortants.

Nous avons ensuite étudié par analyse spectroscopique le gaz plasmagène, plus précisément la zone du plasma proche de la paroi solide (zone appelée " couche limite "). Enfin, nos mesures ont été effectuées sur la surface même du solide : mesures de la température de la paroi solide, analyses ESCA et de microdureté.

\subsection{ANALYSE Des GaZ.}

4.1.1 Variation du taux de décomposition de $\mathrm{NH}_{3}$. La variation du taux de décomposition de $\mathrm{NH}_{3}$ en fonction de la pression et en présence d'un matériau (Fig. 3) met en évidence soit l'existence d'un phénomène de catalyse (cas des métaux de transition)

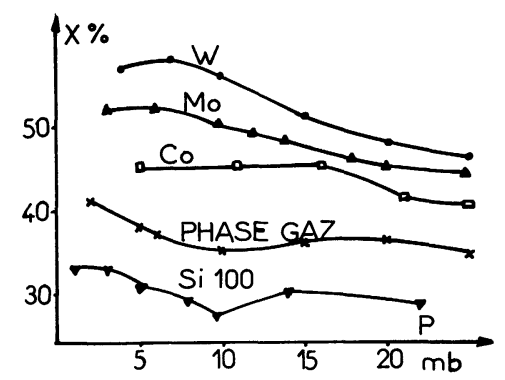

Fig. 3. - Variation du taux de décomposition de $\mathrm{NH}_{3}(X \%)$ en fonction de la pression (mbar) en phase gazeuse et phase hétérogène. $\left(P_{1}=1 \mathrm{~kW} ; D^{0}=0,61 / \mathrm{min}\right.$.)

$\left[\mathrm{NH}_{3}\right.$ decomposition rate $\left(X_{\%}^{\circ}\right)$ against pressure (mbar) in gas phase and heterogeneous phase. $\left(P_{\mathrm{I}}=1 \mathrm{~kW}, D^{0}=\right.$ $0.6 \mathrm{l} / \mathrm{min}$.).] soit l'existence d'un phénomène d'inhibition (cas du semi-conducteur).

Pour les métaux catalyseurs, dans les mêmes conditions opératoires (pression, puissance induite, débit), la valeur du taux de décomposition de $\mathrm{NH}_{3}$ varie selon la nature du métal avec des écarts allant jusqu'à $15 \%$. Il s'agit donc d'un phénomène de chimisorption dissociative et non pas d'une simple absorption physique [10].

Quelle que soit la nature du métal catalyseur le processus catalytique est favorisé par une faible pression, à savoir une forte température vibrationnelle [8].

4.1.2 Analyse spectroscopique de la couche limite. Le profil de la température de vibration de $\mathrm{N}_{2}\left(\mathrm{C}^{3} \Pi_{\mathrm{u}}\right)$ dans le réacteur à plasma en fonction de la nature du solide introduit (Fig. 4) met en évidence l'existence d'une couche limite où la répartition énergétique est différente de celle du coeur du plasma.

Dans cette couche limite (zone frontière entre le solide et le plasma) on observe soit une forte augmentation de la température vibrationnelle (cas des métaux de transition), soit une chute de la température vibrationnelle (cas du silicium mono $\langle 100\rangle$ ) (Fig. 4).

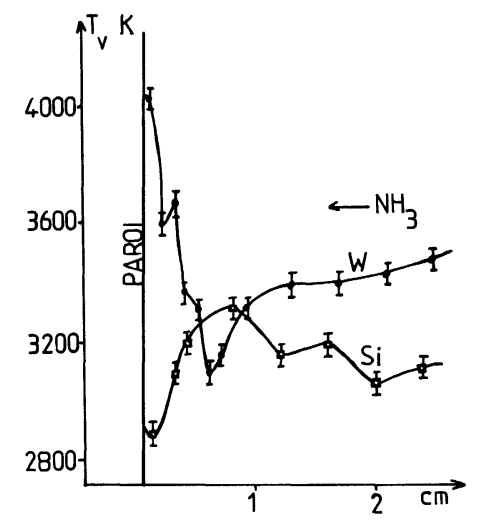

Fig. 4. - Profils de la température vibrationnelle (K) dans un plasma de $\mathrm{NH}_{3}$ en présence d'une surface solide. Tungstène $(\mathrm{W})$ : matériau catalyseur. Silicium $(\mathrm{Si})$ : matériau inhibiteur. $\left(P=5 \mathrm{mbar}, P_{\mathrm{I}}=1 \mathrm{~kW}, D^{0}=0,6 \mathrm{l} / \mathrm{min}\right.$. $)$

[Vibrational temperature (K) profiles in $\mathrm{NH}_{3}$ plasma in presence of a solid surface. Tungsten $(\mathrm{W})$ : catalyst. Silicon $(\mathrm{Si})$ : inhibitor. $\left(P=5 \mathrm{mbar}, P_{1}=1 \mathrm{~kW}, D^{0}=0.61 / \mathrm{min}\right.$.).]

Pour les métaux de transition (catalyseurs) l'augmentation de la température vibrationnelle de $\mathrm{N}_{2}\left(\mathrm{C}^{3} \Pi_{\mathrm{u}}\right)$ varie selon la nature du solide (Fig. 5). De plus, en présence d'un métal de transition, la température vibrationnelle du cour du plasma est plus élevée qu'en son absence (Fig. 6).

Dans le cas du matériau semi-conducteur (inhibiteur) la chute observée pour la température vibrationnelle de $\mathrm{N}_{2}\left(\mathrm{C}^{3} \Pi_{\mathrm{u}}\right)$ est plus forte que celle produite par une paroi de trempe refroidie à l'eau et la température vibrationnelle du cour du plasma est plus faible en présence de ce matériau qu'en son absence (Fig. 7). 


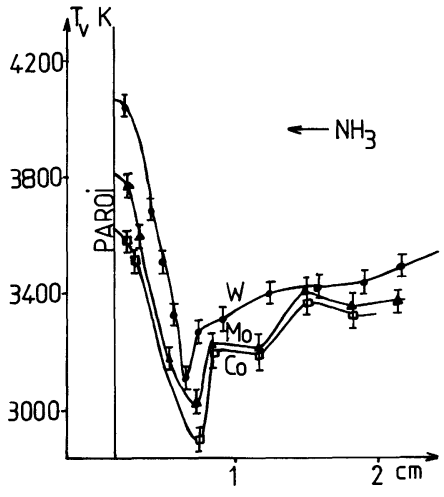

Fig. 5. - Profils de la température vibrationnelle (K) dans un plasma de $\mathrm{NH}_{3}$ selon la nature du matériau catalyseur (W, Mo, Co). $\left(P=5 \mathrm{mbar}, P_{\mathrm{I}}=1 \mathrm{~kW}, D^{0}=0,6 \mathrm{l} / \mathrm{min}\right.$.)

[Vibrational temperature $(\mathrm{K})$ profiles in a $\mathrm{NH}_{3}$ plasma according to the nature of the catalyst $(\mathrm{W}, \mathrm{Mo}, \mathrm{Co}) .(P=$ $5 \mathrm{mbar}, P_{\mathrm{I}}=1 \mathrm{~kW}, D^{0}=0.6 \mathrm{l} / \mathrm{min}$.).]

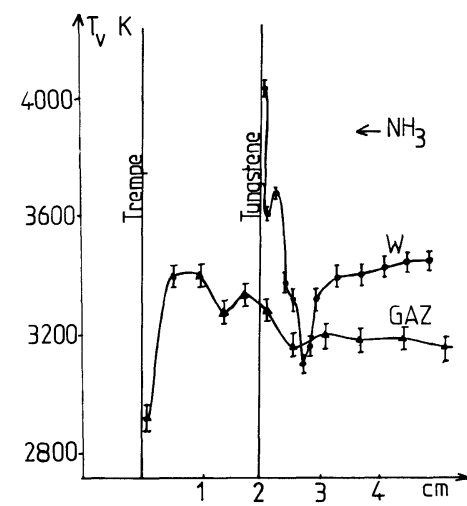

Fig. 6. - Profils de la température vibrationnelle (K) dans un plasma de $\mathrm{NH}_{3}$ en présence d'un matériau catalyseur (W) et en son absence (phase gaz). $\left(P=5 \mathrm{mbar}, P_{\mathrm{I}}=\right.$ $1 \mathrm{~kW}, D^{0}=0,6 \mathrm{l} / \mathrm{min}$.)

[Vibrational temperature $(\mathrm{K})$ profiles in a $\mathbf{N H}_{3}$ plasma in presence of a catalyst $(\mathrm{W})$ and in its absence (gas phase). $\left(P=5 \mathrm{mbar}, P_{\mathrm{I}}=1 \mathrm{~kW}, D^{0}=0.6 \mathrm{l} / \mathrm{min}\right.$.).]

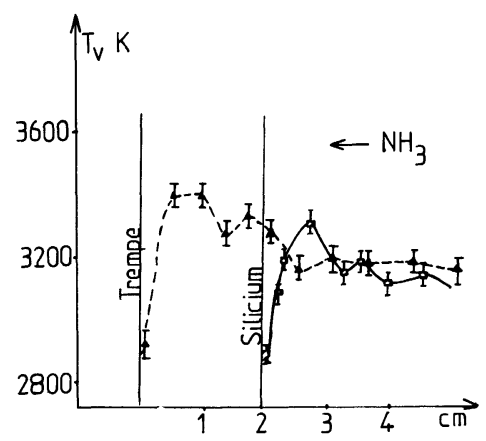

Fig. 7a. - Profils de la température vibrationnelle (K) dans un plasma de $\mathrm{NH}_{3}$, en présence d'un matériau inhibiteur (Si) et en son absence (phase gaz). $\left(P=5 \mathrm{mbar}, P_{\mathrm{I}}=\right.$ $1 \mathrm{~kW}, D^{0}=0,6 \mathrm{l} / \mathrm{min}$.)

[Vibrational temperature (K) profiles in a $\mathrm{NH}_{3}$ plasma, in presence of an inhibitor ( $\mathrm{Si}$ ) and in its absence (gas phase). $\left(P=5 \mathrm{mbar}, P_{\mathrm{I}}=1 \mathrm{~kW}, D^{0}=0.6 \mathrm{l} / \mathrm{min}\right.$. $)$.]

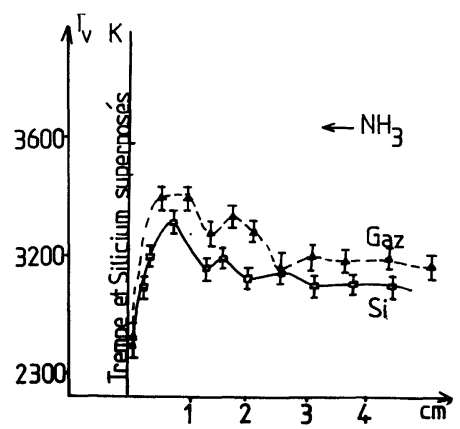

Fig. $7 b$. - Variation de la température vibrationnelle provoquée par une paroi refroidie à l'eau ou par une plaque de silicium, à une même distance de la surface. (Plasma $\mathrm{NH}_{3}$, $P=5 \mathrm{mbar}, P_{\mathrm{I}}=1 \mathrm{~kW}, D^{0}=0,61 / \mathrm{min}$.)

[Variation of the vibrational temperature due to a cooled water surface or to a silicon surface, at the same distance from the surface. (Plasma $\mathrm{NH}_{3}, P=5 \mathrm{mbar}, P_{\mathrm{I}}=1 \mathrm{~kW}$, $D^{0}=0.61 / \mathrm{min}$.).]

\subsection{ANALYSE DE LA SURFACE SOLIDE.}

4.2.1 Variation de la température du solide. - La figure 8 représente la variation de la température de la plaque en fonction de la pression et de la nature du solide introduit. Ce résultat met en évidence l'existence d'un transfert énergétique entre le gaz et le solide. En effet, les solides introduits dans le réacteur dans les mêmes conditions opératoires (pression, puissance induite, débit) s'échauffent différemment et présentent entre eux des différences allant jusqu'à $350{ }^{\circ} \mathrm{C}$ (W-Si).

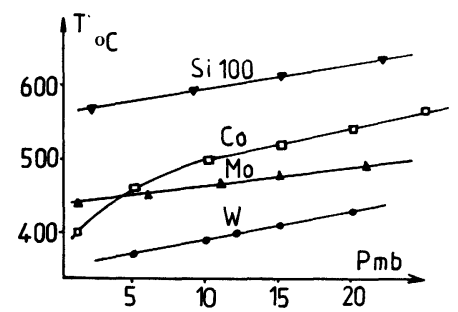

Fig. 8. - Variation de la température des plaques $\left({ }^{\circ} \mathrm{C}\right)$ en fonction de la pression (mbar). (Plasma $\mathrm{NH}_{3}, P_{\mathrm{I}}=1 \mathrm{~kW}$, $D^{0}=0,61 / \mathrm{min}$.)

[Temperatures of the solid $\left({ }^{\circ} \mathrm{C}\right)$ against pressure (mbar). (Plasma $\mathrm{NH}_{3}, P_{\mathrm{I}}=1 \mathrm{~kW}, D^{0}=0.6 \mathrm{l} / \mathrm{min}$.). $]$

4.2.2 Analyse ESCA des surfaces solides. - L'analyse ESCA d'échantillons (W, Mo, $\mathrm{Si}\langle 100\rangle)$ avant et après leur introduction dans le réacteur, met en évidence sans équivoque la formation d'un nitrure lors du traitement.

La figure 9 représente le spectre global d'un échantillon de $\mathrm{W}$ avant traitement. Les pics observés sont respectivement les suivants $O_{1 s}$ (oxyde), $C_{1 s}$ (référence) et $\mathrm{W}_{4 \mathrm{D}}$ (métal). 


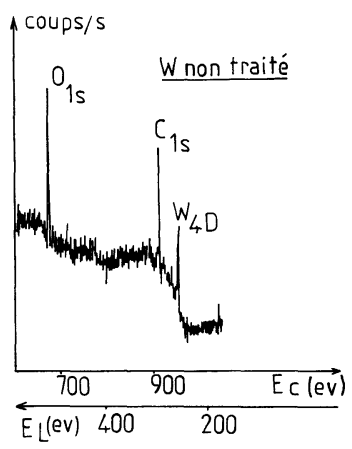

Fig. 9. - Spectre global ESCA d'un échantillon de tungstène $(\mathrm{W})$ non traité.

[ESCA spectrum of a piece of tungsten before treatment.]

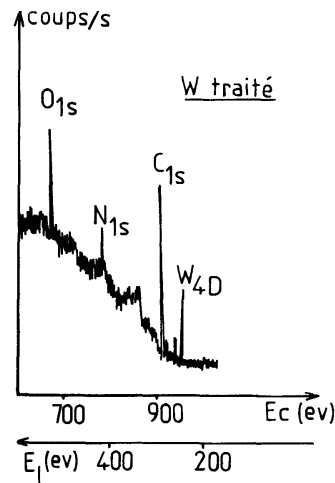

Fig. 10. - Spectre global ESCA d'un échantillon de tungstène $(\mathrm{W})$ traité. (Plasma $\mathrm{NH}_{3}, P=5 \mathrm{mbar}, P_{\mathrm{I}}=1 \mathrm{~kW}$, $D^{0}=0,6 \mathrm{l} / \mathrm{min}$., durée du traitement : $1 \mathrm{~h} \mathrm{30}$.)

[ESCA spectrum of a piece of tungsten after treatment. (Plasma $\mathrm{NH}_{3}, P=5 \mathrm{mbar}, P_{\mathrm{I}}=1 \mathrm{~kW}, D^{0}=0.6 \mathrm{l} / \mathrm{min}$., $1 \mathrm{~h} 30 \mathrm{~min}$.).]

Tableaux I et II. - Valeur des énergies de liaison des nitrures.

TABLEAU I

\section{TABLEAU II}

\begin{tabular}{|c|c|c|c|}
\hline Espèces & $\begin{array}{c}\text { WN } \\
\text { standard }\end{array}$ & $\begin{array}{c}\mathrm{W}_{2} \mathrm{~N} \\
\text { standard }\end{array}$ & $\begin{array}{c}\text { W traité en plasma NH } \\
P=5 \mathrm{mbar}, P_{\mathrm{I}}=1 \mathrm{~kW}, D^{0}=0,6 \mathrm{l} / \mathrm{min} . \\
\text { Durée } 1 \mathrm{~h} 30\end{array}$ \\
\hline \multirow[t]{2}{*}{$\begin{array}{l}W_{4 D 5 / 2} \\
N_{1 s}\end{array}$} & $\begin{array}{l}244,5 \mathrm{eV} \\
397 \mathrm{eV}\end{array}$ & $\begin{array}{l}247,5 \mathrm{eV} \\
397 \mathrm{eV}\end{array}$ & $\begin{array}{l}244,2 \mathrm{eV} \\
396,0 \mathrm{eV}\end{array}$ \\
\hline & $\begin{array}{c}\text { MoN } \\
\text { standard }\end{array}$ & $\begin{array}{c}\mathrm{Mo}_{2} \mathrm{~N} \\
\text { standard }\end{array}$ & $\begin{array}{c}\text { Mo traité en plasma NH } \\
P=5 \mathrm{mbar}, P_{1}=2 \mathrm{~kW}, D^{0}=0,6 \mathrm{l} / \mathrm{min} . \\
\text { Durée } 1 \mathrm{~h} 30\end{array}$ \\
\hline \multirow[t]{2}{*}{$\begin{array}{l}\mathrm{Mo}_{3 \mathrm{D} \mathrm{5/2}} \\
\mathrm{N}_{1 \mathrm{~s}}\end{array}$} & $\begin{array}{l}228,4 \mathrm{eV} \\
396,5 \mathrm{eV}\end{array}$ & $\begin{array}{l}232,6 \mathrm{eV} \\
396,5 \mathrm{eV}\end{array}$ & $\begin{array}{l}228,6 \mathrm{eV} \\
396,5 \mathrm{eV}\end{array}$ \\
\hline & \multicolumn{2}{|c|}{$\mathrm{Si}_{3} \mathrm{~N}_{4}$ standard } & $\begin{array}{c}\text { Si traité en plasma } \mathrm{NH}_{3} \\
P=5 \mathrm{mbar}, P_{\mathrm{I}}=1 \mathrm{~kW}, D^{0}=0,61 / \mathrm{min} . \\
\text { Durée } 30 \mathrm{~min} .\end{array}$ \\
\hline $\begin{array}{l}\mathrm{Si}_{2 \mathrm{~s}} \\
\mathrm{~N}_{1 \mathrm{~s}}\end{array}$ & \multicolumn{2}{|c|}{$\begin{array}{l}151,8 \mathrm{eV} \\
396,5 \mathrm{eV}\end{array}$} & $\begin{array}{l}152,1 \mathrm{eV} \\
397,1 \mathrm{eV}\end{array}$ \\
\hline
\end{tabular}

La figure 10 représente le spectre global d'un échantillon de $\mathrm{W}$ traité en phase plasma $\mathrm{NH}_{3}$ pendant $1 \mathrm{~h} 30 \mathrm{~min}$. $\left(P=5 \mathrm{mbar}, P_{\mathrm{I}}=1 \mathrm{~kW}, D^{0}=0,6 \mathrm{l} / \mathrm{min}\right.$. $)$. Outre les pics déjà cités $\left(U_{1 s}, C_{1 s}, W_{4 D}\right)$ on voit apparaître un pic d'azote $\mathrm{N}_{1 \mathrm{~s}}$ pour une énergie de liaison égale à $396 \mathrm{eV}$.

Des résultats analogues ont été obtenus pour des échantillons de Mo et $\mathrm{Si}\langle 100\rangle$; il y a donc bien formation d'un nitrure lors du traitement en phase plasma.

Afin d'identifier les nitrures formés nous avons basé notre étude sur les énergies de liaison.

Le tableau I donne les énergies de liaison des électrons des atomes $\mathrm{N}_{1 \mathrm{~s}}, \mathrm{~W}_{4 \mathrm{D} 5 / 2}, \mathrm{Mo}_{3 \mathrm{D} 5 / 2}$ et $\mathrm{Si}_{2 \mathrm{~s}}$ pour les nitrures standards suivants : $\mathrm{WN}, \mathrm{W}_{2} \mathrm{~N}, \mathrm{MoN}$, $\mathrm{Mo}_{2} \mathrm{~N}, \mathrm{Si}_{3} \mathrm{~N}_{4}$.

Une simple comparaison de ces énergies de liaison avec celles des nitrures formés en phase plasma (Tableau II) permet de mettre en évidence la stoéchiométrie des nitrures formés en phase plasma c'est-àdire WN, $\mathrm{MoN}$ et $\mathrm{Si}_{3} \mathrm{~N}_{4}$.

Le calcul de la surface des pics rend compte de l'évolution de la couche de nitrure en fonction du

Tableau III. - Intensités relatives des pics pour différents temps de traitement. Echantillon de $\mathrm{Mo}\left(\mathrm{gaz} \mathrm{NH}_{3}\right.$, $P=5 \mathrm{mbar}, P_{\mathrm{I}}=1 \mathrm{~kW}, D^{0}=0,6 \mathrm{l} / \mathrm{min}$.).

\begin{tabular}{|c|cccc|}
\hline \multirow{2}{*}{$\mathrm{N} /$ métal } & \multicolumn{4}{|c|}{ Durée du traitement } \\
& $1 \mathrm{~h}$ & $1 \mathrm{~h} 30$ & $2 \mathrm{~h}$ & $3 \mathrm{~h}$ \\
\hline$\frac{\mathrm{N}_{1 \mathrm{~s}}}{\mathrm{Mo}_{3 \mathrm{D} 5 / 2}}$ & 1,7 & 1,9 & 2,1 & 2,3 \\
\hline
\end{tabular}


temps par la mesure des rapports $\mathrm{N} /$ métal (Tableau III).

4.2.3 Microdureté. - L'étude de la microdureté permet de rendre compte de la cinétique de la nitruration en suivant l'évolution de la dureté d'échantillons, (W, Mo) en fonction de la durée de traitement.

Un premier travail a été effectué sur des échantillons de W, Mo non traités et traités pendant $1 \mathrm{~h} 30 \mathrm{~min}$. $\left(P=5 \mathrm{mbar}, P_{\mathrm{I}}=1 \mathrm{~kW}, D^{0}=0,6 \mathrm{l} / \mathrm{min}\right.$.). Cette approche montre, pour une charge constante de $100 \mathrm{~g}$, une augmentation de la dureté de l'échantillon traité, augmentation qui est en relation avec la formation du nitrure (Tableau IV).

Tableau IV. - Variation de la microdureté Vickers en $\mathrm{kg} / \mathrm{mm}^{2}$ en fonction de la charge $(\mathrm{g})$, et en fonction de la profondeur de pénétration du diamant $(\mu \mathrm{m})$.

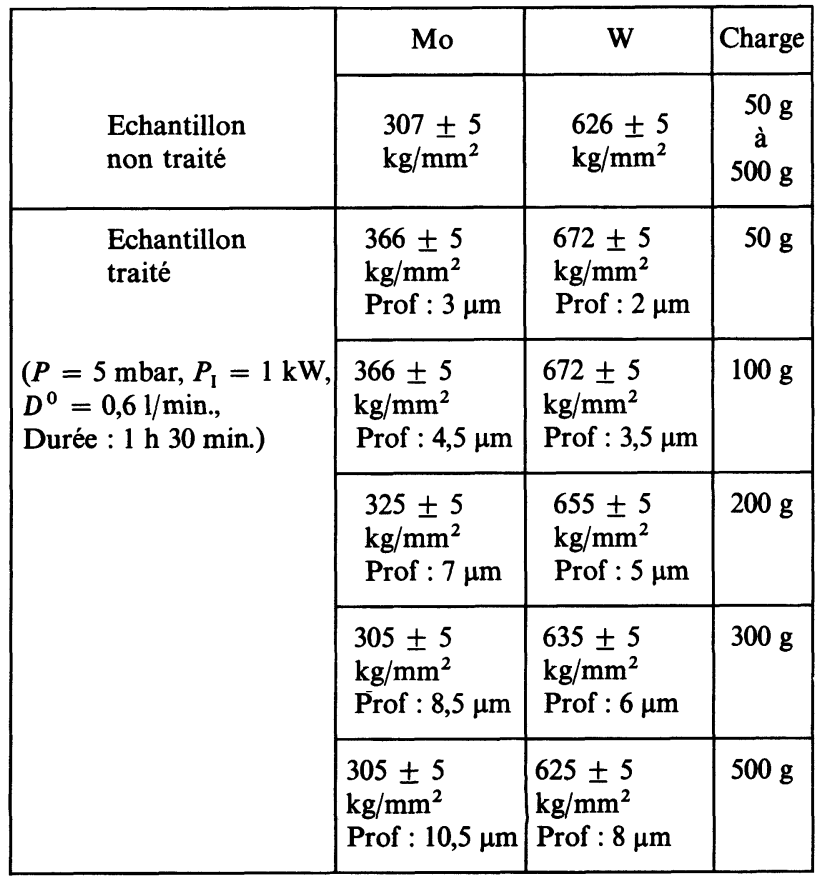

La variation des valeurs de dureté de ces échantillons avec la charge, c'est-à-dire avec la profondeur de pénétration du diamant, permet de déterminer approximativement : $5 \mu$, l'épaisseur de la couche formée (Tableau IV et Fig. 11a).

Une étude systématique a ensuite été effectuée sur des échantillons de Mo pour des durées croissantes de traitement $\left(P=5 \mathrm{mbar}, P_{\mathrm{I}}=1 \mathrm{~kW}, D^{0}=0,6 \mathrm{l} / \mathrm{min}\right.$., charge constante $=100 \mathrm{~g}$ ) (Tableau V).

Ces valeurs de microdureté permettent de dégager une loi de formation du nitrure en log temps (Fig. 11b). Ce résultat est en accord avec les conclusions d'autres

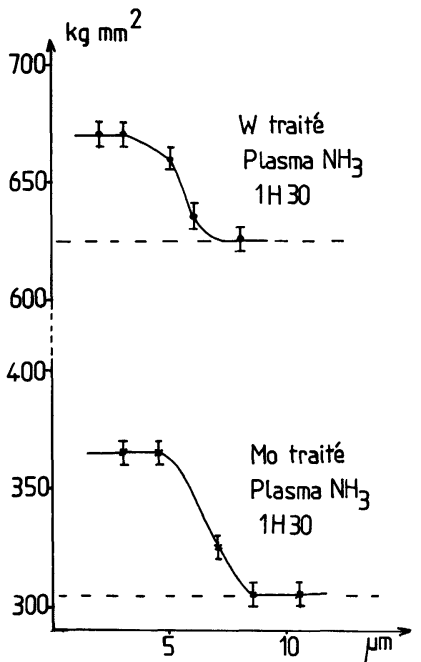

Fig. 11a. - Variation de la microdureté $\mathrm{HV}\left(\mathrm{kg} / \mathrm{mm}^{2}\right)$ d'échantillons de $\mathrm{W}$ et Mo traités, en fonction de la profondeur de pénétration $(\mu)$. (Traitement des échantillons : plasma $\mathrm{NH}_{3}$, durée $=1$ h 30 min., $P=5$ mbar, $P_{\mathrm{I}}=1 \mathrm{~kW}$, $D^{0}=0,61 / \mathrm{min}$.) 307 et $625 \mathrm{~kg} / \mathrm{mm}^{2}$ : valeurs respectives d'échantillons de Mo et $\mathrm{W}$ non traités.

[Vickers microhardness $\left(\mathrm{kg} / \mathrm{mm}^{2}\right)$ of Mo and $\mathrm{W}$ treated pieces against penetration depth (micron). (Treatment : Plasma $\mathrm{NH}_{3}, P=5 \mathrm{mbar}, P_{\mathrm{I}}=1 \mathrm{~kW}, D^{0}=0.6 \mathrm{l} / \mathrm{min}$, time of exposure $=1 \mathrm{~h} 30 \mathrm{~min}$.) 307 and $625 \mathrm{~kg} / \mathrm{mm}^{2}$ : values of untreated Mo and W respectively.]

travaux, effectués sur l'oxydation des solides [11-13] qui donnent pour loi générale une relation logarithmique entre l'épaisseur de la couche formée et le temps d'exposition $(x=a \log (1+b t))$.

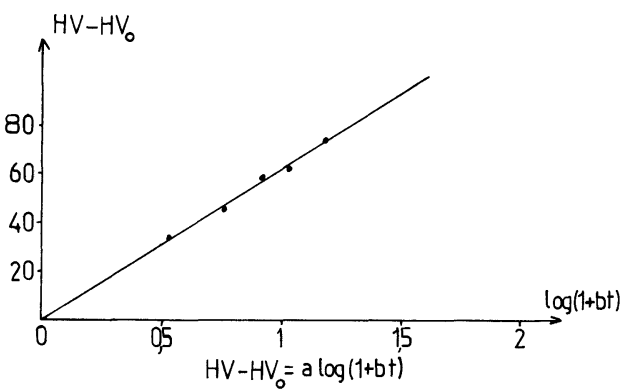

Fig. 11b. - Variation de la microdureté $\mathrm{HV}\left(\mathrm{kg} / \mathrm{mm}^{2}\right)$ d'un échantillon de molybdène en fonction de la durée de traitement (s) $\mathrm{HV}-\mathrm{HV}_{0}=a \log (1+b t)$; où $\mathrm{HV}_{0}$ valeur pour $t=0$ (échantillon non traité). (Plasma $\mathrm{NH}_{3}, P=$ $5 \mathrm{mbar}, P_{\mathrm{I}}=1 \mathrm{~kW}, D^{0}=0,6 \mathrm{l} / \mathrm{min}$.)

[Vickers microhardness $\left(\mathrm{kg} / \mathrm{mm}^{2}\right.$ ) of a piece of molybdenum against time of exposure (s). $\mathrm{HV}-\mathrm{HV}_{0}=a \log (1+b t)$ where $\mathrm{HV}_{0}$ hardness at $t=0 \mathrm{~s}$ (before treatment). (Plasma $\mathrm{NH}_{3}, P=5 \mathrm{mbar}, P_{1}=1 \mathrm{~kW}, D^{0}=0.6 \mathrm{l} / \mathrm{min}$.).]

Tableau V. - Variations de la microdureté Vickers avec la durée du traitement (charge constante : $100 \mathrm{~g}$ ).

\begin{tabular}{|c|c|c|c|c|c|c|}
\hline & Mo non traité & $\begin{array}{c}\text { Mo traité } \\
30 \mathrm{~min} .\end{array}$ & $\begin{array}{c}\text { Mo traité } \\
1 \mathrm{~h}\end{array}$ & $\begin{array}{c}\text { Mo traité } \\
1 \mathrm{~h} 30 \mathrm{~min} .\end{array}$ & $\begin{array}{c}\text { Mo traité } \\
2 \mathrm{~h}\end{array}$ & $\begin{array}{c}\text { Mo traité } \\
3 \mathrm{~h}\end{array}$ \\
\cline { 2 - 7 } $\mathrm{HV}\left(\mathrm{kg} / \mathrm{mm}^{2}\right)$ & $307 \pm 5$ & $341 \pm 5$ & $352 \pm 5$ & $366 \pm 5$ & $370 \pm 5$ & $381 \pm 5$ \\
\hline
\end{tabular}




\section{Interprétation.}

Les résultats expérimentaux mettent en évidence une modification du taux de décomposition de $\mathrm{NH}_{3}$ et de la répartition énergétique du plasma due à l'introduction d'un matériau solide dans le réacteur. De plus, un transfert de matière entre le gaz et le solide s'effectue, ce qui conduit à la nitruration du matériau.

Des résultats cités ci-dessus se dégagent deux faits marquants. En premier lieu, le phénomène de catalyse est lié à une forte augmentation de la température vibrationnelle de $\mathrm{N}_{2}\left(\mathrm{C}^{3} \Pi_{\mathrm{u}}\right)$ au niveau de la paroi (couche limite) et à une température vibrationnelle élevée dans le cour du plasma tandis que le phénomène d'inhibition est lié à une chute de la température vibrationnelle de $\mathrm{N}_{2}\left(\mathrm{C}^{3} \Pi_{\mathrm{u}}\right)$ au niveau de la paroi et à une faible température vibrationnelle dans le cour du plasma.

En second lieu, l'échauffement présenté par un matériau catalyseur est beaucoup plus faible que celui d'un matériau inhibiteur pour les mêmes conditions opératoires.

L'existence de cette corrélation entre la réactivité chimique et la répartition énergétique nous a conduit dans un premier temps à étudier séparément le phénomène de catalyse et celui d'inhibition afin de les relier aux transferts énergétiques. Une fois cette relation déterminée, nous avons étudié le transfert de matière qui a lieu quel que soit le phénomène (catalyse ou inhibition) observé afin de le relier aux processus déjà cités et d'approfondir la connaissance des mécanismes réactionnels des interactions plasma-surface.

5.1 Phénomène de CATAlyse. - Dans le processus catalytique observé en présence d'un métal de transition, la température de vibration de $\mathrm{N}_{2}\left(\mathrm{C}^{3} \Pi_{\mathrm{u}}\right)$ semble jouer un rôle prépondérant puisqu'elle augmente fortement au niveau de la paroi, de façon moindre dans le cour du plasma et prend des valeurs différentes selon la nature du métal. L'existence de ce paramètre et de son influence nous a conduit à scinder le processus de catalyse en deux étapes : l'étape d'adsorption et celle de recombinaison-désorption.

5.1.1 Rôle de la température vibrationnelle dans l'adsorption. - Un système hors d'équilibre tel que le milieu plasma basse pression est un milieu dans lequel l'énergie n'est pas répartie de façon homogène entre les différents degrés de liberté de la molécule.

L'influence de l'excitation préférentielle d'un mode d'énergie a été étudiée dans le cas de réactions en phase gazeuse par l'équipe de Polanyi [1-3]. Leurs expériences ont montré que, d'une façon générale, les réactions exothermiques sont favorisées par une énergie translationnelle élevée des réactifs tandis que les réactions endothermiques le sont par une énergie vibrationnelle élevée. Les produits de la réaction sont alors vibrationnellement excités dans le premier cas, et translationnellement excités dans le second cas.

L'étude de la réaction de décomposition de $\mathrm{NH}_{3}$ en phase gaz a confirmé les résultats obtenus par Polanyi et al. En effet, cette réaction endothermique est favorisée en phase gaz par une diminution de pression, à savoir une forte énergie vibrationnelle des réactifs, les transferts vibration $\rightarrow$ translation ou vibration $\rightarrow$ rotation étant freinés [8].

Le problème est donc de savoir dans quelle mesure l'excitation préférentielle d'un mode d'énergie, et plus précisément du mode d'énergie vibrationnelle, peut jouer un rôle dans le cas de réactions hétérogènes.

A partir de modèles mathématiques simulant la collision d'une molécule gazeuse avec une surface solide, Mac Creery et Wolken ont étudié les dynamiques d'adsorption [4]. Les calculs montrent qu'une valeur élevée de la température de vibration favorise l'étape d'adsorption dans le cas où la surface d'énergie potentielle, du système gaz solide, est répulsive ou faiblement répulsive et si la température translationnelle est inférieure à $1500 \mathrm{~K}$. C'est le cas de la chimisorption présentant une énergie d'activation (Fig. 12). Citons pour exemples l'adsorption de $\mathrm{N}_{2}$ sur $\mathrm{Fe}$ ou $\mathrm{H}_{2}$ sur W.

Par contre, la température de vibration est sans effet sur la dynamique d'adsorption pour une surface d'énergie potentielle attractive qui correspond à une adsorption spontanée et présente un puits de potentiel. (Fig. 12). C'est le cas de l'adsorption de $\mathrm{H}_{2}$ sur $\mathrm{Cu}$.
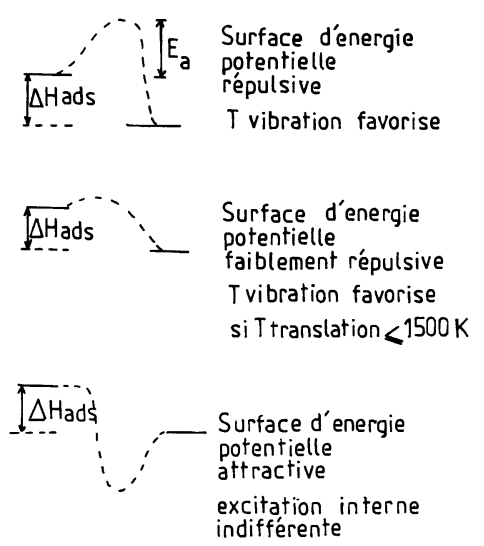

Fig. 12. - Rôle de l'excitation interne dans l'adsorption, d'après Wolken [4].

[Role of the internal excitation in the adsorption step, according to Wolken [4].]

Nos résultats confirment les conclusions de Mac Creery et Wolken. En effet, d'une part le taux de décomposition de $\mathrm{NH}_{3}$ en phase hétérogène, pour un catalyseur donné, augmente avec l'écart à l'équilibre $\alpha$ du plasma $\left(\alpha=T_{\text {vib }}-T_{\text {rot }} / T_{M}\right.$ avec $T_{M}=T_{\text {vib }}+$ $T_{\text {rot }}$, Fig. 13). D'autre part, la comparaison de l'action des différents catalyseurs montre que le taux de décomposition de $\mathrm{NH}_{3}(X \%)$ et la température vibrationnelle $\left(T_{\text {vib }}\right.$ de $\left.\mathrm{N}_{2}\left(\mathrm{C}^{3} \Pi_{\mathrm{u}}\right)\right)$ à une pression donnée varient dans le même sens, c'est-à-dire :

$$
X \%{ }^{\circ}>{ }^{\circ} \text { мо }>X_{\%} \% \text { co (Fig. 3) }
$$


et

$$
T_{\text {vib }} \mathrm{W}>T_{\text {vib }} \text { Mo }>T_{\text {vib }} \text { Co, (Fig. 5). }
$$

D'autres auteurs travaillant sur l'influence de l'excitation par laser ont aussi constaté une forte augmentation du rendement catalytique lorsqu'on irradie le gaz c'est-à-dire lorsqu'on augmente son excitation vibrationnelle [14-17].

Citons par exemple, la réaction $\mathrm{Si}_{-} \mathrm{SF}_{6}$ induite par un laser pulsé à $\mathrm{CO}_{2}$ et qui mène à la formation de molécules de $\mathrm{SiF}_{4}$, même à basse température, lorsque la fréquence du laser est en résonance avec celle des excitations vibrationnelles des molécules $\mathrm{SF}_{6}$ adsorbées [17].

En conclusion, lors de l'introduction d'une plaque métallique dans un réacteur à plasma basse pression il y a excitation préférentielle du mode vibrationnel favorisant ainsi la dynamique d'adsorption à savoir le rendement catalytique.

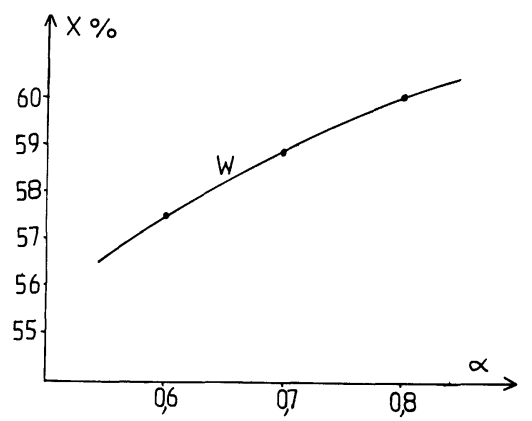

Fig. 13. - Variation du taux de décomposition de $\mathrm{NH}_{3}(X \%)$ en fonction de l'écart à l'équilibre $(\alpha)$ du plasma. $\left(\alpha=\frac{T_{\mathrm{vib}}-T_{\text {rot }}}{T_{\mathrm{vib}}+T_{\text {rot }}} ; 0<\alpha<1\right)$ en présence d'une plaque de tungstène $(W)$.

$\left[\mathrm{NH}_{3}\right.$ decomposition rate against equilibrium divergence $\alpha$, in presence of a piece of tungsten $\left(\alpha=T_{\text {vib }}-T_{\text {rot }} / T_{M}\right.$, $\left.T_{\mathrm{M}}=T_{\text {vib }}+T_{\text {rot }} ; 0<\alpha<1\right)$.]

5.1.2 Etape de recombinaison-désorption. - L'étape de recombinaison-désorption est une étape exothermique qui libère de l'énergie. Par exemple, lors de la désorption d'une molécule de $\mathrm{N}_{2}, 1,7 \mathrm{eV}$ sont libérés dans le cas du tungstène [10].

Le problème posé est donc de savoir comment se répartit cette énergie excédentaire, si elle est captée préférentiellement par le gaz ou le solide. L'étude bibliographique fournit deux types de renseignements :

a) Les calculs théoriques de Wolken pour les dynamiques de recombinaison-désorption [5] prévoient que si la surface d'énergie potentielle gaz-solıde est de type répulsif (c'est-à-dire avec une énergie d'activation élevée) ou endothermique (Fig. 14), la réaction de recombinaison-désorption ne peut avoir lieu quel que soit le mode d'excitation favorisé. Par contre, si la surface d'énergie potentielle est attractive, c'est-à-dire pour des réactions de surfaces exothermiques présentant une barrière d'activation faible ou nulle (Fig. 14),
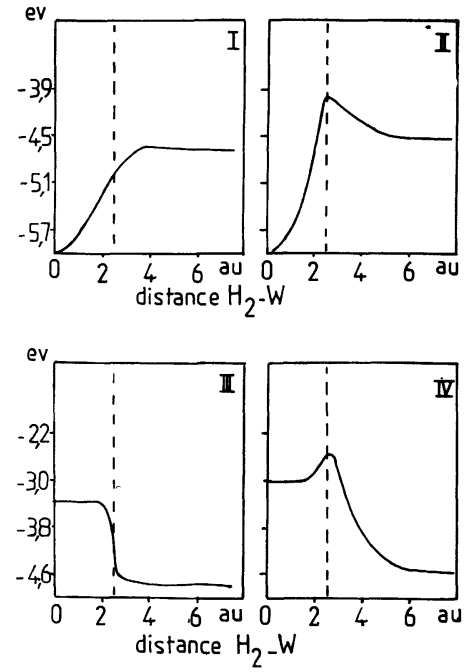

Fig. 14. - Profil énergétique des réactions de recombinaison selon la nature des surfaces d'énergie potentielle d'après Wolken [5]. I, II, surfaces d'énergie potentielle répulsive. III, IV, surfaces d'énergie potentielle attractive.

[Energy profiles of recombination reactions along the reaction paths for different potential surfaces according to Wolken [5]. I, II, repulsive potential surfaces. III, IV, attractive potential surfaces.]

la réaction de recombinaison-désorption a lieu et les molécules produites sont vibrationnellement et rotationnellement excitées. L'excitation se fait préférentiellement, sur le mode vibrationnel pour des molécules désorbées symétriques. Pour des molécules désorbées présentant deux atomes de masse différente, les divers modes d'excitation ont tendance à s'équilibrer [5].

b) Les expériences effectuées par Halpern et Rosner sur la recombinaison-désorption d'atomes d'azote sur différents métaux [9] prévoient que la majeure partie de l'énergie de recombinaison $Q$ sera captée par la molécule d'azote désorbée. Ils ont mesuré expérimentalement un coefficient d'accommodation $\beta$ qui représente la fraction maximale d'énergie de recombinaison qui peut être captée par le métal et qui est définie telle que :

$$
\beta=\frac{Q}{\Delta n(D / 2)},
$$

où $Q=$ énergie de recombinaison,

$\Delta n=$ nombre atomes d'azote/unité de temps,

$D=$ énergie de dissociation moléculaire.

La variation du coefficient $\beta$ avec la température du métal montre qu'il existe deux régions (Fig. 15). Une région de " basse température » où $\beta$ est généralement faible (exemple dans le cas du tungstène $\beta<0,1$ si $T<1600 \mathrm{~K}$ ) et où la plupart de l'excès énergétique est prise par le gaz; et une région de " haute température " où les valeurs de $\beta$ sont élevées (exemple dans le cas du tungstène, $\beta$ augmente de 0,1 à 1 lorsque la température varie de $1600 \mathrm{~K}$ à 


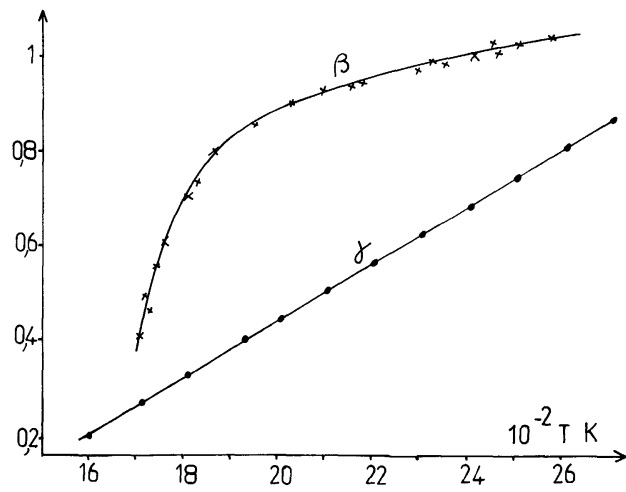

Fig. 15. - Variation du coefficient d'accommodation $\beta$ et de la probabilité de recombinaison atomique $\gamma$ en fonction de la température du solide $(K)$ pour la recombinaison des atomes d'azote sur le tungstène $(\mathrm{W})$ d'après Halpern et Rosner [9].

[Temperature dependence of the chemical energy accommodation coefficient $\beta$ and atom recombination probability $\gamma$ for the $\mathrm{N}$ atom/tungsten system according to Halpern et Rosner [9].]

$2400 \mathrm{~K}$ ) et où par conséquent la part d'énergie captée par le solide n'est pas négligeable.

La valeur de $\beta$ varie aussi selon la nature du système gaz-solide (exemple à $T=1000 \mathrm{~K} \beta$ pour l'azote sur cobalt est égal à 1 tandis que $\beta$ pour l'azote sur tungstène est inférieur à 0,1 (Figs. 15 et 16)).

Dans notre réacteur à plasma basse pression; la température de translation est relativement faible (800 à $1000 \mathrm{~K}$ ) ainsi que les températures des métaux catalyseurs $(600$ à $800 \mathrm{~K})$.

Du point de vue du partage de l'énergie entre gaz et solide, nous nous trouvons donc dans la région dite de « basse température " où l'excès d'énergie est capté préférentiellement par le gaz. De plus, la nature symétrique de la molécule désorbée $\mathrm{N}_{2}$ doit conduire d'après Wolken [5] à une forme d'excitation vibrationnelle pour $\mathrm{N}_{2}$.

Nos résultats confirment le fait qu'il y a partage de l'énergie excédentaire entre le gaz et le solide. En effet, d'une part nous avons mis en évidence l'existence d'une couche limite (Fig. 5) présentant une forte excitation vibrationnelle $\mathrm{N}_{2}\left(\mathrm{C}^{3} \Pi_{\mathrm{u}}\right)$ due à la part importante d'énergie captée par la molécule désorbée et canalisée vers son mode vibrationnel en raison de sa symétrie [5]. D'autre part, la mesure des températures des métaux catalyseurs (auxquels aucune chaleur extérieure n'est fournie) met en évidence un échauffement du catalyseur.

De plus les écarts de température présentés par les différents métaux sont en accord avec les coefficients d'accommodation $\beta$ calculés par Halpern et Rosner [9] $\left(T_{\mathrm{Co}}>T_{\mathrm{w}}\right.$ Fig. 8 et $\beta_{\mathrm{Co}}>\beta_{\mathrm{w}}$ Figs. 15 et 16$)$. Par conséquent, l'échauffement du solide est en partie dû à l'excès d'énergie de la réaction de surface. L'autre facteur qui contribue à l'échauffement du solide est le transfert " physique " gaz solide. Nous avons essayé de garder ce facteur constant en maintenant à des

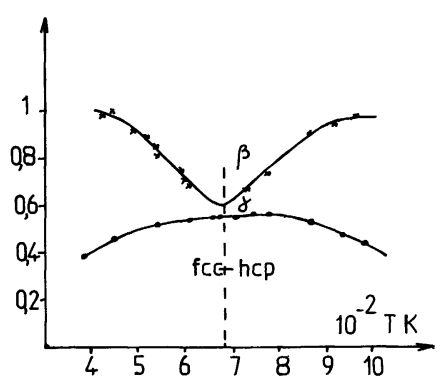

Fig. 16. - Variation du coefficient d'accommodation $\beta$ et de la probabilité de recombinaison atomique $\gamma$ en fonction de la température du solide $(\mathrm{K})$ pour la recombinaison des atomes d'azote sur le cobalt $(\mathrm{Co})$ d'après Halpern et Rosner [9].

[Temperature dependence of the chemical energy accommodation coefficient $\beta$ and atom recombination probability $\gamma$ for the $\mathbf{N}$ atom/cobalt system according to Halpern et Rosner [9].]

valeurs fixes la puissance induite, le débit et la pression du gaz. $\left(P_{\mathrm{I}}=1 \mathrm{~kW}, D^{0}=0,6 \mathrm{l} / \mathrm{min} ., P=5 \mathrm{mbar}\right.$, surface de la plaque $50 \times 70 \mathrm{~mm}^{2}$ ).

5.1.3 Mécanisme d'activation rétroactif. - La synthèse des deux étapes de la réaction hétérogène : réactions d'adsorption et de recombinaison-désorption, reliée aux faits expérimentaux suivants : l'activité catalytique d'un métal est liée à une forte température vibrationnelle de $\mathrm{N}_{2}\left(\mathrm{C}^{3} \Pi_{\mathrm{u}}\right)$ et à un faible échauffement du métal, nous a permis de dégager un mécanisme d'activation énergétique rétroactif.

L'étude de la dynamique de recombinaison désorption a montré qu'il y avait libération d'une énergie excédentaire. Cette énergie se répartit entre le solide qui s'échauffe et les molécules de $\mathrm{N}_{2}$ désorbées qui s'excitent vibrationnellement. Cette augmentation de la température vibrationnelle au niveau de la paroi se transmet par transferts vibration $\left(\mathrm{N}_{2}\right)$-vibration $\left(\mathrm{NH}_{3}\right)$ aux molécules de $\mathrm{NH}_{3}$ incidentes favorisant ainsi leur adsorption sur le métal (Fig. 17).

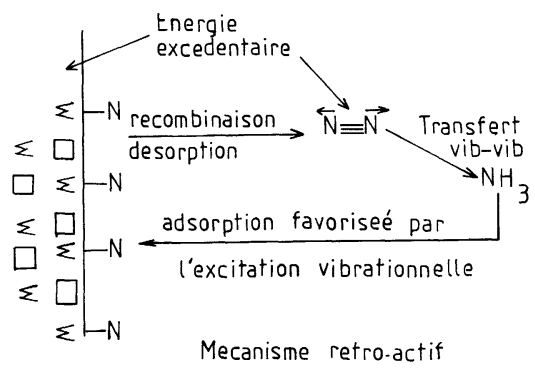

Fig. 17. - Mécanisme d'activation énergétique rétro-actif.

[Feed-back mechanism.]

Ce mécanisme est corroboré par l'ensemble des courbes 3,5 et 8 . En effet, lors du partage de l'énergie excédentaire, plus la part captée par le solide est faible (donc son échauffement moindre), plus la part prise 
par les molécules de $\mathrm{N}_{2}$ désorbées sera élevée. Par conséquent, l'augmentation vibrationnelle des molécules de $\mathrm{N}_{2}$ désorbées en sera d'autant plus importante, les transferts vibration $\left(\mathrm{N}_{2}\right)$-vibration $\left(\mathrm{NH}_{3}\right)$ facilités, l'adsorption des molécules de $\mathrm{NH}_{3}$ favorisée et le taux de décomposition de $\mathrm{NH}_{3}$ en sera d'autant plus élevé.

Cette hiérarchie des événements est bien respectée puisque :

$$
\begin{array}{lll}
T \text { sol }(\mathrm{W})<T \text { sol }(\mathrm{Mo})<T \text { sol }(\mathrm{Co}), & \text { (Fig. 8) } \\
T \text { vib }(\mathrm{W})>T \operatorname{vib}(\mathrm{Mo})>T \text { vib }(\mathrm{Co}), & \text { (Fig. 5) } \\
X_{\%}^{\circ}(\mathrm{W})>X \%(\mathrm{Mo})>X \%(\mathrm{Co}) . & \text { (Fig. 3) } .
\end{array}
$$

Le phénomène de catalyse et les transferts énergétiques sont donc intimement liés. En effet, l'activité catalytique d'un métal pour la réaction de décomposition de $\mathrm{NH}_{3}$ est directement reliée à son aptitude à augmenter l'excitation vibrationnelle à son voisinage et par transferts dans le cour du plasma.

5.2 PHÉNOMÈNE D'INHIBITION. - L'introduction d'une plaque de silicium mono $\langle 100\rangle$ dans le réacteur à plasma $\mathrm{NH}_{3}$ basse pression entraîne une baisse du taux de décomposition de $10 \%$ par rapport à la phase homogène (Fig. 3). De plus, cette plaque de silicium, bien que présentant un échauffement très élevé (Fig. 8) (environ $900 \mathrm{~K}$ ), provoque une chute de la température vibrationnelle de $\mathrm{N}_{2}\left(\mathrm{C}^{3} \Pi_{\mathrm{u}}\right)$ beaucoup plus importante que celle entraînée par la trempe (Fig. 7).

Cela est dû au fait qu'en présence de silicium, la réaction de chimisorption n'a pas lieu. De plus, au lieu d'être le support d'une réaction pouvant fournir de l'énergie au gaz (recombinaison-désorption), il joue le rôle d'un absorbeur d'énergie beaucoup plus efficace qu'une paroi refroidie à l'eau (Figs. $7 a$ et $7 b$ ). De ce fait il présente un échauffement élevé et thermalise le plasma à son voisinage. Cette baisse de l'énergie se répercute dans le coeur du plasma et inhibe de ce fait la réaction clef endothermique $\mathrm{NH}_{3} \rightarrow \mathrm{NH}+\mathrm{H}_{2}$ qui est contrôlée par l'excitation vibrationnelle en phase gazeuse.

En conclusion, l'activité inhibitrice d'un matériau pour la réaction de décomposition de $\mathrm{NH}_{3}$ est liée à son aptitude à diminuer l'excitation vibrationnelle ầ son voisinage et dans le cœur du plasma.

5.3 Transfert DE MATière. - Les études du phénomène de catalyse et d'inhibition ont démontré que l'activité catalytique ou inhibitrice d'un matériau est liée à son aptitude à modifier la répartition énergétique du plasma, plus précisément à modifier l'excitation vibrationnelle qui est le mode d'excitation qui régit la réaction. En fait, il est probable que le réel catalyseur ou inhibiteur soit le nitrure et non pas le matériau lui-même. En effet, les travaux effectués par l'équipe de Boudart [18] sur l'activation des liaisons $\mathrm{N}-\mathrm{N}, \mathrm{H}-\mathrm{H}$ par certains nitrures ont mis en évidence une relation entre le pouvoir catalytique d'un matériau et sa nitruration in situ pour la réaction de décomposi- tion ou de synthèse de $\mathrm{NH}_{3}$. Pour la synthèse de $\mathrm{NH}_{3}$ à $1 \mathrm{~atm}$, en présence d'une poudre de Mo employée comme catalyseur, il a été démontré que le taux de réaction en fonction du temps croît très rapidement puis atteint une valeur stationnaire dès que deux couches de $\mathrm{Mo}_{2} \mathrm{~N}$ sont formées.

Ensuite, le phénomène de nitruration se poursuit tandis que le rendement de la réaction reste constant en fonction du temps.

Ces travaux rendent donc compte de la supériorité du pouvoir catalytique du nitrure par rapport au métal.

Dans notre cas nous n'avons pas mis en évidence une variation du taux de décomposition de $\mathrm{NH}_{3}$ lors de la formation des premières couches de nitrure, en raison de la rapidité des réactions de nitruration en phase plasma (la formation d'une monocouche se produit en $1 \mu$ s à 1 torr). Nos mesures de CVP ont été effectuées alors que l'état stationnaire était déjà atteint.

Le fait que ce soit le nitrure et non le métal lui-même qui catalyse la réaction de décomposition de $\mathrm{NH}_{3}$ entraîne que l'activité catalytique d'un matériau sera directement reliée à son aptitude à la nitruration. On retrouve expérimentalement cette relation, en effet dans un plasma de $\mathrm{NH}_{3}$ la comparaison des analyses effectuées avec W et Mo montre que c'est le meilleur catalyseur de la réaction de décomposition de $\mathrm{NH}_{3}$ qui présente le taux de fixation d'azote le plus élevé (Fig. 3, Tableau VI). Cela est dû au fait qu'une des étapes fondamentales de la nitruration est la chimisorption dissociative.

Tableau VI. - Echantillons traités en gaz $\mathrm{NH}_{3}$. Durée $1 \mathrm{~h} 30, P=5 \mathrm{mbar}, P_{1}=1 \mathrm{~kW}, D^{0}=0,61 / \mathrm{min}$.

\begin{tabular}{|l|c|c|c|}
\hline \multirow{2}{*}{$\begin{array}{c}\text { Proprièté } \\
\text { Taux fixation } \\
\text { N/espèce }\end{array}$} & W & \multicolumn{3}{|c|}{ Echantillon } \\
\cline { 2 - 4 } & catalyseur & catalyseur & inhibiteur \\
\hline
\end{tabular}

L'analogie qui existe entre l'oxydation des surfaces et la nitruration en phase plasma $\mathrm{NH}_{3}$ semble confirmer ce fait. En effet, pour l'oxydation des solides, de nombreux auteurs [19-26] ont démontré qu'à basse température ou basse pression, la formation de la couche d'oxyde passe par une couche chimisorbée bien définie. Or, dans nos propres expériences sur les métaux de transition en plasma $\mathrm{NH}_{3}$, nous avons les mêmes phénomènes c'est-à-dire chimisorption dissociative et incorporation d'atomes dans le solide. De plus, l'équipe de Namba [24] a mis en évidence pour le système $\mathrm{Mg}-\mathrm{O}_{2}$ une augmentation du taux de chimisorption lors de la formation des deux premières couches de $\mathrm{MgO}$, augmentation analogue à celle observée par Boudart [18] pour le système Mo-NH . Enfin, nous avons trouvé une relation cinétique de formation de nitrure en plasma de $\mathrm{NH}_{3}$, analogue 
à celle déterminée pour l'oxydation des solides $(x \propto$ $\log (b t+1))$. Ces analogies nous laissent suggérer que la plupart des mécanismes proposés pour l'oxydation restent valables pour la nitruration en phase plasma $\mathrm{NH}_{3}$. Or, actuellement, deux mécanismes de croissance de la couche d'oxyde (après formation des deux premières couches) sont proposés. Mott et Fehlner suggèrent [11] que l'étape déterminante est le passage, par effet tunnel ou thermoionique, d'un électron du métal à travers la couche d'oxyde jusqu'à certains niveaux accepteurs des atomes $\mathbf{O}$ adsorbés. Ceci entraînerait la formation d'ions oxygène à la surface et d'ions métalliques à l'interface, par conséquent la création d'un champ électrique qui favoriserait l'incorporation des atomes (electric field assisted). Il semble que les processus d'anodisation en phase plasma relèvent de ce mécanisme [27].

Eley et Wilkinson [12], ainsi que Kirk et Huber [13] supposent que les atomes d'oxygène chimisorbés sont incorporés grâce à un mécanisme d'échange avec les atomes du réseau.

Ces deux mécanismes rendent compte de l'importance de la couche chimisorbée pour les réactions de surface. Ceci explique pourquoi le W, qui est le métal qui favorise le plus l'adsorption grâce au mécanisme d'activation énẻrgétique déjà évoqué (cf. 5.1.3) présente un taux de fixation d'azote beaucoup plus élevé que celui du Mo alors que la solubilité de l'azote est plus faible dans le W que dans le Mo [28](Tableau VII).

Tableau VII. - Solubilité de l'azote à 1 atmosphère. Comparaison W-Mo [28].

\begin{tabular}{|c|c|c|c|c|}
\hline \multirow{2}{*}{$T^{\circ} \mathrm{C}$} & \multicolumn{2}{|c|}{ Tungstène } & \multicolumn{2}{|c|}{ Molybdène } \\
\hline & $\% \mathrm{~N}$ (pds) & $\% \mathrm{~N}$ (at) & $\% \mathrm{~N}$ (pds) & $\% \mathrm{~N}$ (at) \\
\hline \begin{tabular}{ll|}
1 & 200 \\
1 & 600 \\
2 & 000 \\
2 & 400
\end{tabular} & $\begin{array}{l}0,13 \times 10^{-5} \\
0,19 \times 10^{-4} \\
0,11 \times 10^{-3} \\
0,38 \times 10^{-3}\end{array}$ & 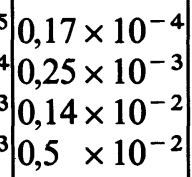 & $\left\{\begin{array}{r}1,05 \times 10^{-3} \\
4,3 \times 10^{-3} \\
1,05 \times 10^{-2} \\
2 \quad \times 10^{-2}\end{array}\right.$ & $\begin{array}{l}14,7 \times 10^{-3} \\
60,2 \times 10^{-3} \\
14,7 \times 10^{-2} \\
28 \times 10^{-2}\end{array}$ \\
\hline
\end{tabular}

Le mécanisme de nitruration est donc régi pour les métaux de transition, catalyseurs de la réaction de décomposition de $\mathrm{NH}_{3}$, par l'étape de chimisorption dissociative et non pas par l'étape diffusionnelle; tandis que pour le silicium, il n'y a pas phénomène de chimisorption dissociative (comme l'ont montré les résultats d'analyse CVP et spectroscopique). De ce fait, la nitruration ne peut se faire que par diffusion gazeuse d'atomes $\mathbf{N}$ (dont le nombre est peu élevé) ce qui explique le bas taux de fixation d'azote observé.

\section{Conclusion.}

Au cours de ce travail nous avons étudié les mécanismes réactionnels des interactions plasma surface pour la réaction de décomposition de $\mathrm{NH}_{3}$ et nous avons mis en évidence les relations existant entre les processus de catalyse ou d'inhibition, les transferts énergétiques, les transferts de matière et le caractère hors d'équilibre d'un plasma basse pression.

Un matériau catalyseur provoque une forte augmentation de la température vibrationnelle de $\mathrm{N}_{2}\left(\mathrm{C}^{3} \Pi_{\mathrm{u}}\right)$ à son voisinage, s'échauffe peu et présente un taux de fixation d'azote élevé; tandis qu'un matériau inhibiteur entraîne une chute de la température vibrationnelle près de sa paroi, s'échauffe beaucoup et présente un faible taux de fixation d'azote.

Pour le phénomène catalytique, il s'agit d'un processus de chimisorption dissociative. L'activité du catalyseur est liée à son aptitude à renvoyer l'énergie vibrationnelle, ce qui augmente le caractère hors d'équilibre du plasma, favorise l'étape d'adsorption des molécules donc le rendement de la réaction et facilite la nitruration du matériau.

Pour le processus d'inhibition, il s'agit d'une absorption par le matériau de toute forme d'énergie. De ce fait, le matériau inhibiteur présente un fort échauffement et thermalise le plasma, ce qui inhibe la réaction clef de la décomposition de $\mathrm{NH}_{3}$, réaction endothermique favorisée par le caractère hors d'équilibre du plasma. De même la réaction de nitruration est défavorisée puisque dans ce cas l'incorporation des atomes $\mathrm{N}$ ne peut se faire que par diffusion gazeuse et ne passe pas par une étape de chimisorption dissociative qui est l'étape déterminante du processus.

En conclusion, l'introduction d'un matériau dans un plasma basse pression modifie la répartition énergétique du plasma. Le processus catalytique dépendra du caractère hors équilibre du plasma, en particulier de l'aptitude du procédé à transférer l'énergie sur l'état vibrationnel dont dépendent les réactions chimiques des espèces (Fig. 18).

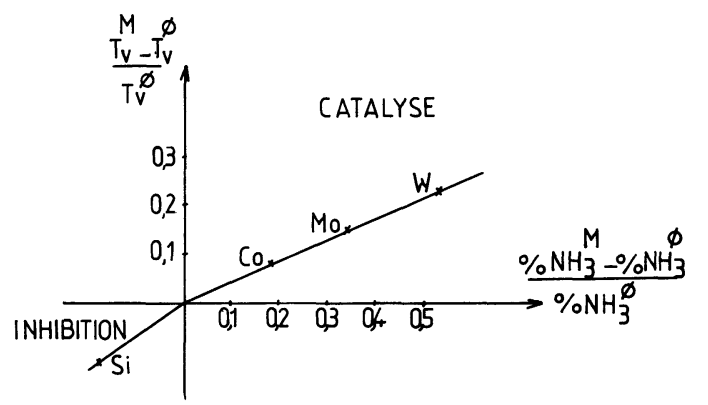

Fig. 18. - Contribution de la modification de l'énergie vibrationnelle d'un plasma de $\mathrm{NH}_{3}$ par un matériau, à la catalyse ou à l'inhibition. $T_{\mathrm{v}}^{\mathrm{M}}:$ Température vibrationnelle d'un plasma de $\mathrm{NH}_{3}$ en présence d'un matériau. $T_{\mathrm{V}}^{\phi}$ : Température vibrationnelle d'un plasma de $\mathrm{NH}_{3}$ en phase gaz. $\% \mathrm{NH}_{3}^{\mathrm{M}}$ : Taux de décomposition de $\mathrm{NH}_{3}$ en présence d'un matériau. \% $\mathrm{NH}_{3}^{\phi}$ : Taux de décomposition de $\mathrm{NH}_{3}$ en phase gaz.

[Contribution of the vibrational energy modification of a plasma by a solid, to the catalyst or the inhibitor effect. $T_{\mathrm{V}}^{\mathrm{M}}$ : Vibrational temperature of a $\mathrm{NH}_{3}$ plasma in presence of a solid. $T_{\mathrm{V}}^{\phi}$ : Vibrational temperature of a $\mathrm{NH}_{3}$ plasma in gas phase. $\% \mathrm{NH}_{3}^{\mathrm{M}}: \mathrm{NH}_{3}$ decomposition rate in presence of a solid. $\% \mathrm{NH}_{3}^{\phi}: \mathrm{NH}_{3}$ decomposition rate in gas phase.] 
Au contraire, une thermalisation du plasma c'est-àdire une distribution énergétique régulière élimine une partie des états vibrationnels et favorise le processus de chauffage du matériau, c'est-à-dire inhibe les réactions chimiques endothermiques.

Soulignons que cette démarche conduit à la recherche du concept nouveau dans de nombreuses applications qu'il s'agisse de procédés de gravure sous plasma, de procédé de cémentation de matériau ou de la recherche de gaz diélectrique pour les procédés d'isolement haute tension.

\section{Bibliographie}

[1] Polanyi, J. C., J. Chem. Soc. Faraday. Dis. (1975)

[2] Polanyi, J. C., Wong, W. H., J. Chem. Phys. 51 (4) (1969) 1439.

[3] Polanyi, J. C., Acc. Chem. Res. 5 (1972) 161.

[4] McCreery, J., Wolken, G., J. Chem. Phys. 65 (1976) 1310.

[5] McCreery, J., Wolken, G., J. Chem. Phys. 67 (1977) 2551.

[6] Rapakoulias, D., Amouroux, J., Bergougnan, M. P., GiCQuel, A., Proceeding of the 3rd symposium on plasma processing, 160 th meeting of the electrochemical Society Denver USA (edited by Dielman and Frieser (USA), vol. 82-6, 1982, p. 88-113.

[7] Rapakoulias, D., Amouroux, J., Bergougnan, M. P., Gicquel, A., Proceeding of the 3rd symposium on plasma processing, 160th meeting of the electrochemical Society Denver (USA) (edited by Dielman and Frieser (USA)), vol. 82-6. 1982, p. 114-137.

[8] Rapakoulias, D., Amouroux, J., Domange, M., Rev. Phys. Appl. 16 (1981) 343.

[9] Halpern, B., Rosner, D. E., J. Chem. Soc. Faraday. Trans. I-60 (1977) 1883.

[10] Rapakoulias, D., Amouroux, J., Bergougnan, M. P., Gicquel, A., Rev. Phys. Appl. 17 (1982) 95-101.

[11] Fehner, F. P., Mott, N. F., Oxid. Met. 2 (1970) 59.

[12] Eley, D. D., Wilkinson, P. R., Proc. R. Soc. London A 254 (1960) 327.

[13] KIRK, C. T. Jr, Huber, E. E. Jr, Surf. Sci. 9 (1968) 217.

[14] Umstead, M. E., Lin, M. C., J. Phys. Chem. 82 (1978) 2047.
[15] Lin, C. T., Atvars, D. Z., J. Chem. Phys. 68 (9) (1978) 4233.

[16] UMSTEADT, M. E. et al., Opt. Eng. 19 (1980) 94.

[17] Chuang, T. J., Vibrations at surface edited by R. Caudeno, J. M. Gilles and A. A. Lucas (Plenum New York) 1982, p. 573.

[18] Boudart, M., Oyama, S. T., Leclerce, L., Stud. Surf. Sci. catal. 7 (1981) 578-90.

[19] BRUNDLE, C. R., Aspects of the kinetics and dynamics of surface reactions, edited by Uzi Landman (American Institute of Physics, New York) 1980, p. 57.

[20] Ling, P. T., Miller, J. N., Pianetta, P., Weissman, D. L., LindaU, I., SPICER, W. E., J. Vac. Sci. Technol. 21 (1982) 47.

[21] Hofman, P., Wyrobisch, W., Bradshaw, A., Surf. Sci. 80 (1979) 344.

[22] Orent, T. W., BaDER, S. D., Surf. Sci. 115 (1982) 323.

[23] Hsin-Kuei Hu, Wayne Rabalais, J., Surf. Sci. 107 (1981) 376.

[24] Namba, H., Darville, J., Gilles, J. M., Surf. Sci. 108 (1981) 446.

[25] Matsuyama, T., Ignatiev, A., Surf. Sci. 102 (1981) 18.

[26] Castner, D. G., Somorjai, G. A., Appl. Surf. Sci. 6 (1980) 29.

[27] O'Halnon, J. F., J. Vac. Sci. Technol. 7 (1970) 330.

[28] Hansen, M., Constitution of binary alloys, 2nd edition (edited by Mac Graw-Hill book company Inc.) 1958. 